\title{
Organocatalyzed Asymmetric Reactions via Microwave Activation
}

\section{Sarah Mossé and Alexandre Alexakis*}

Department of Organic Chemistry, University of Geneva, 30, quai Ernest Ansermet, CH-1211 Geneva 4, Switzerland

\section{Supporting Information}

\section{Table of Contents}

General remarks

S1

Microwave irradiation experiments

Aldol reactions with chiral separations

Conjugate additions with chiral separations

Diels-Alder reaction with chiral separation

${ }^{1} \mathrm{H}$ spectra of 3a-e, 6a-c, and $\mathbf{1 0}$

Examples of temperature, pressure and microwave power profiles

General Remarks. Concerning ${ }^{1} \mathrm{H}(400 \mathrm{MHz})$ and ${ }^{13} \mathrm{C}(100 \mathrm{MHz}) \mathrm{NMR}$ spectra, chemical shift $(\delta)$ are given in $\mathrm{ppm}$ relative to tetramethylsilane $(0 \mathrm{ppm})$. Multiplicity is indicated as follows : s (singlet), d (doublet), t (triplet), q (quartet), m (multiplet), dd (doublet of doublet), br s (broad singlet). Coupling constants are reported in Hz. Optical rotations were measured at $25^{\circ} \mathrm{C}$ in a $10 \mathrm{~cm}$ cell in the stated solvent ; $[\alpha]_{\mathrm{D}}$ values are given in $10^{-1}$ deg.cm $\mathrm{g}^{-1}$ (concentration c given as $\mathrm{g} / 100 \mathrm{~mL}$ ). Enantiomeric excesses were determined by chiral Super Fluid Chromatography (SFC), with appropriated program using a gradient of methanol or by chiral Gas Chromatography (GC), with appropriated program using a gradient of temperature. Temperature programs are described as follows: initial temperature $\left({ }^{\circ} \mathrm{C}\right)$ - initial time $(\mathrm{min})-$ temperature gradient $\left({ }^{\circ} \mathrm{C} / \mathrm{min}\right)$ - final temperature $\left({ }^{\circ} \mathrm{C}\right)$; retention times $\left(\mathrm{R}_{\mathrm{T}}\right)$ are given in min. Flash chromatography was performed using silicagel 32-63 $\mu \mathrm{m}, 60 \AA$.

Evolution of reaction was followed by TLC. Benzaldehyde 2c and isobutyraldehyde 4c were freshly distilled before using. Cyclopentadiene monomer 7 was freshly used after cracking. $(S, S)$ - $N$-Isopropyl-2,2'-bipyrrolidine (iPBP) was prepared as described in literature procedure. $^{1}$ (5S)-5-Benzyl-2,2,3-trimethylimidazolidin-4-one hydrochloride $\mathbf{9}$ was prepared as

\footnotetext{
${ }^{1}$ O. Andrey, A. Alexakis, A. Tomassini, G. Bernardinelli, Adv. Synth. Catal. 2004, 346, 1147.
} 
described in literature. ${ }^{2}$ All other reagents and solvents were purchased from commercial suppliers and used as received.

\section{Microwave irradiation experiments.}

Microwave-assisted reactions were carried out using a focused microwave unit (Biotage Initiator $^{\mathrm{TM}}$ ). The instrument consists of a continuous focused microwave power delivery system with operator selectable power output from 0-300 W. In all experiments, a constant power was applied to ensure reproducibility. Reactions were performed in glass vessels (2-5 $\mathrm{mL}$ ) sealed with a septum. Pressure measurement is accomplished by a non-invasive sensor integrated into the cavity lid, which measures the deformation of the Teflon seal of the vessels (maximum 20 bar). Temperature controlled is achieved by means of an IR sensor and the indicated temperature corresponds to the maximal temperature reached during each experiment. The specified reaction time corresponds to the total irradiation time. Efficient cooling is accomplished by means of a pressurized air ( with a pressure of 5 bar) during the entire experiment.

\footnotetext{
${ }^{2}$ Ahrendt, K. A.; Borths, C. J.; MacMillan, D. W. C. J. Am. Chem. Soc. 2000, 122, 4243.
} 


\section{Aldol reactions}

General procedure 1 under microwave irradiation. In a 2-5 mL vessel were introduced Lproline, DMSO $(2.4 \mathrm{~mL})$ and finally acetone $1(20 \mathrm{vol} \%, 0.6 \mathrm{~mL})$ and the mixture was stirred for $15 \mathrm{~min}$ under argon. Aldehyde was added $(0.3 \mathrm{mmol})$ and the vessel was sealed with a septum and placed into the microwave cavity. The microwave source was then turned on. Constant microwave irradiation as well as simultaneous air-cooling ( 5 bar) were used during the entire reaction time. The evolution of the reaction was monitored by TLC. After cooling to room temperature, the mixture was quenched as described in literature procedure ${ }^{3}$ using $\mathrm{NH}_{4} \mathrm{Cl}$ aq. sat. and extracted with EtOAc $(2 \mathrm{x})$. The combined organic layers were washed successively with water and brine, dried over $\mathrm{MgSO}_{4}$, filtered, concentrated under reduced pressure and purified by flash column chromatography on silica gel using a mixture of cyclohexane and ethyl acetate.

\footnotetext{
${ }^{3}$ a) List, B.; Lerner, R. A.; Barbas III, C. F. J. Am. Chem. Soc. 2000, 22, 2345. b) Sakthivel, K.; Notz, W.; Bui, T.; Barbas III, C. F. J. Am. Chem. Soc. 2001, 23, 5260.
} 


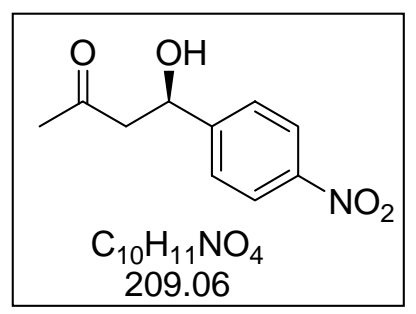

(R)-4-hydroxy-4-(4-nitrophenyl)butan-2-one 3a (Table 2, entry 1): From 4-nitrobenzaldehyde 2a (1 eq., $0.3 \mathrm{mmol}, 45.3 \mathrm{mg}$ ) and Lproline (0.2 eq., $0.06 \mathrm{mmol}, 6.9 \mathrm{mg})$ according to general procedure $1\left(15 \mathrm{~W}, 0 \mathrm{bar}, 35^{\circ} \mathrm{C}\right)$ to give complete conversion after $15 \mathrm{~min}$ and to afford aldol product 3a (43.3 $\mathrm{mg}, 69 \%)$. The enantiomeric excess was determined by chiral SFC (AS-H column, $2 \mathrm{~mL} / \mathrm{min}, 200$ bar, methanol $\left.10 \%-2-1-25 \%, 30^{\circ} \mathrm{C}, R_{\mathrm{t}}: 3.03(R), 3.89(S)\right)$. $[\alpha]_{\mathrm{D}}{ }^{20}=+42.6($ c 1.035 , ee $=70 \%$, $\left.\mathrm{CHCl}_{3}\right)$.

${ }^{1} \mathrm{H}$ NMR $\left(400 \mathrm{MHz}, \mathrm{CDCl}_{3}\right): \delta=8.22-8.20(\mathrm{~d}, \mathrm{~J}=8.60 \mathrm{~Hz}, 2 \mathrm{H}), 7.55-7.52(\mathrm{~d}, \mathrm{~J}=8.84 \mathrm{~Hz}$, $2 \mathrm{H}), 5.28-5.24(\mathrm{~m}, 1 \mathrm{H}), 3.56-3.55(\mathrm{~m}, 1 \mathrm{H}), 2.86-2.84(\mathrm{~d}, 2 \mathrm{H}), 2.22(\mathrm{~s}, 3 \mathrm{H})$. Other spectroscopic data are in agreement with published data. ${ }^{3}$

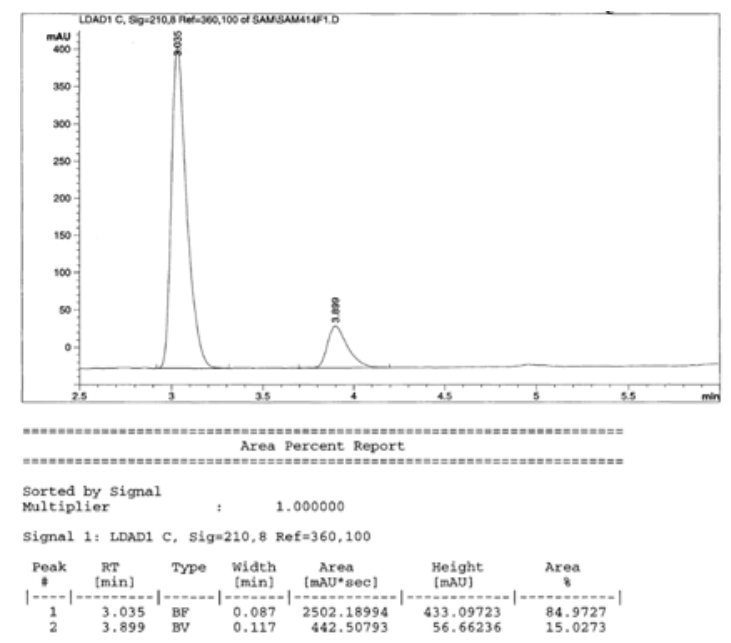


<smiles>CCCCOc1ccc([C@@H](O)CC(C)=O)cc1</smiles>

(R)-4-hydroxy-4-(4-(trifluoromethyl)phenyl)butan-2-one 3b (Table

2, entry 2): From $\alpha, \alpha, \alpha$-trifluoro-p-tolualdehyde $2 \mathbf{b}$ (1 eq., 0.3 mmol, $52.23 \mathrm{mg}$ ) and L-proline (0.2 eq., $0.06 \mathrm{mmol}, 6.9 \mathrm{mg}$ ) according to general procedure $1\left(15 \mathrm{~W}, 0\right.$ bar, $\left.32^{\circ} \mathrm{C}\right)$ to give complete conversion after $15 \mathrm{~min}$ and to afford aldol product $\mathbf{3 b}$ (48.5 mg, 70\%). The enantiomeric excess was determined by chiral SFC (AS-H column, 2 $\mathrm{mL} / \mathrm{min}, 200$ bar, methanol 2\%-2-1-15\%, 30 $\left.{ }^{\circ} \mathrm{C}, R_{\mathrm{t}}: 2.95(R), 3.43(S)\right) .[\alpha]_{\mathrm{D}}{ }^{20}=+32.6(c 1.14$, ee $\left.=70 \%, \mathrm{CHCl}_{3}\right)$.

${ }^{1} \mathrm{H}$ NMR $\left(400 \mathrm{MHz}, \mathrm{CDCl}_{3}\right): \delta=7.62-7.60(\mathrm{~d}, \mathrm{~J}=8.08 \mathrm{~Hz}, 2 \mathrm{H}), 7.49-7.47(\mathrm{~d}, \mathrm{~J}=8.08 \mathrm{~Hz}$, $2 \mathrm{H}), \quad 5.23-5.20(\mathrm{~m}, 1 \mathrm{H}), 3.44-3.43(\mathrm{~m}, 1 \mathrm{H}), 2.85-2.84(\mathrm{~d}, 2 \mathrm{H}), 2.21(\mathrm{~s}, 3 \mathrm{H})$. Other spectroscopic data are in agreement with published data. ${ }^{4}$

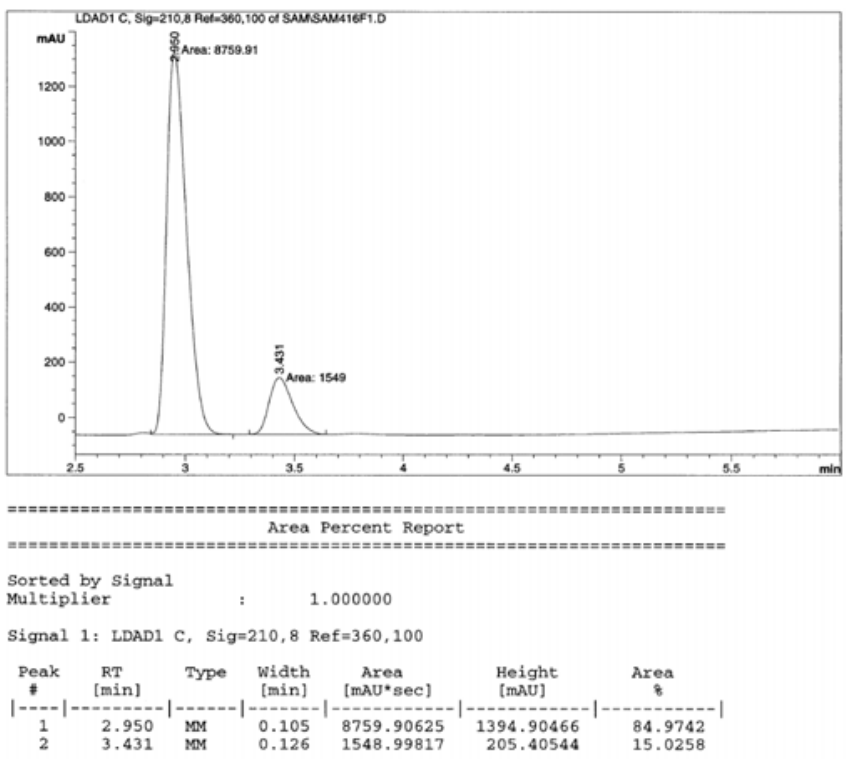

${ }^{4}$ Kotrusz, P.; Kmentová, I.; Gotov, B.; Toma, Š.; Solčániová, E. Chem. Comm. 2002, 2510. 


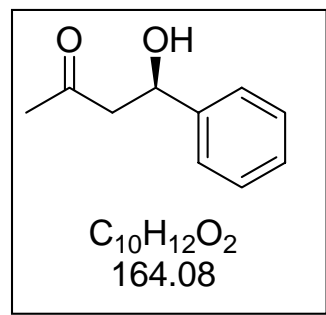

(R)-4-hydroxy-4-phenylbutan-2-one 3c (Table 2, entry 3): From benzaldehyde 2c (1 eq., $0.3 \mathrm{mmol}, 30 \mu \mathrm{L})$ and L-proline (0.3 eq., 0.09 mmol, $10.3 \mathrm{mg}$ ) according to general procedure $1\left(15 \mathrm{~W}, 0\right.$ bar, $\left.31{ }^{\circ} \mathrm{C}\right)$ to give $89 \%$ conversion after $1 \mathrm{~h}$ and to afford aldol product 3c $(34.2$ mg, 70\%). The enantiomeric excess was determined by chiral SFC (OD$\mathrm{H}$ column, $2 \mathrm{~mL} / \mathrm{min}, 200$ bar, methanol 10\%-2-1-25\%, 30 $\left.{ }^{\circ} \mathrm{C}, R_{\mathrm{t}}: 6.60(R), 6.98(S)\right) .[\alpha]_{\mathrm{D}}{ }^{20}=$ $+37.2\left(\right.$ c $\left.0.575, \mathrm{ee}=68 \%, \mathrm{CHCl}_{3}\right)$.

${ }^{1} \mathrm{H}$ NMR $\left(400 \mathrm{MHz}, \mathrm{CDCl}_{3}\right): \delta=7.36-7.30(\mathrm{~m}, 4 \mathrm{H}), 7.29-7.26(\mathrm{~m}, 1 \mathrm{H}), 5.17-5.14(\mathrm{~m}, 1 \mathrm{H})$, $3.29(\mathrm{br}, 1 \mathrm{H}), 2.92-2.79(\mathrm{~m}, 2 \mathrm{H}), 2.19(\mathrm{~s}, 3 \mathrm{H})$. Other spectroscopic data are in agreement with published data. ${ }^{3}$

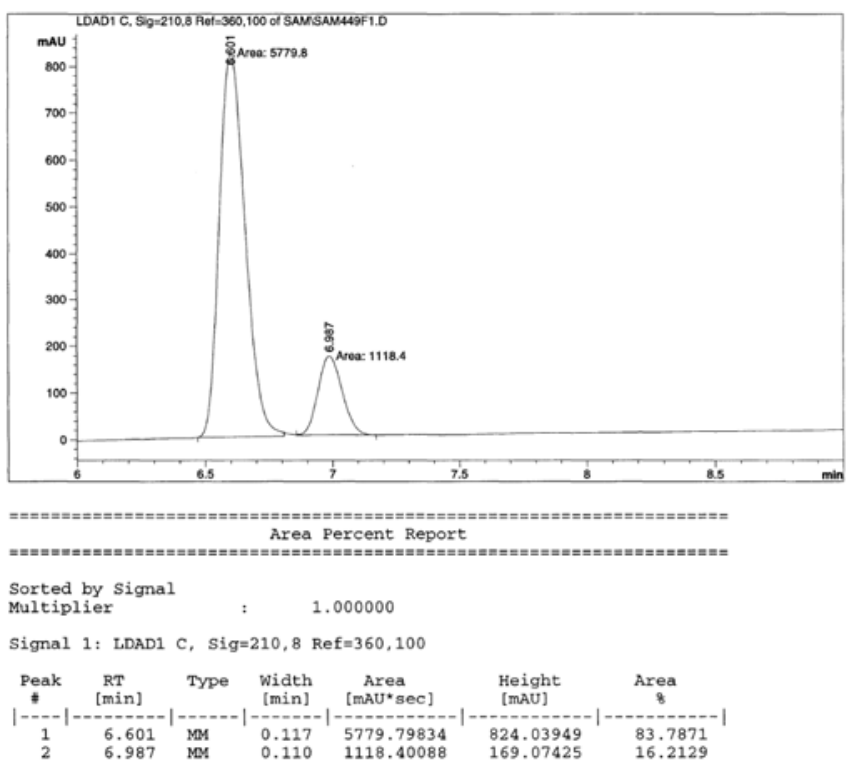


<smiles>CC(=O)CC(O)c1ccc2ccccc2c1</smiles>

(R)-4-hydroxy-4-(naphthalen-2-yl)butan-2-one 3d (Table 2, entry 4): From 2-naphtaldehyde $2 d$ (1 eq., $0.3 \mathrm{mmol}, 46.9 \mathrm{mg}$ ) and Lproline (0.3 eq., $0.09 \mathrm{mmol}, 10.3 \mathrm{mg}$ ) according to general procedure $1\left(15 \mathrm{~W}, 0 \mathrm{bar}, 31^{\circ} \mathrm{C}\right)$ to give $79 \%$ conversion after $1 \mathrm{~h}$ and to afford aldol product 3d (35.9 mg, 56\%). The enantiomeric excess was determined by chiral SFC (AD column, $2 \mathrm{~mL} / \mathrm{min}, 200$ bar, methanol 10\%-2-1$\left.25 \%, 30^{\circ} \mathrm{C}, R_{\mathrm{t}}: 6.94(R), 7.61(S)\right) .[\alpha]_{\mathrm{D}}^{20}=+34.8\left(\right.$ c $\left.0.525, \mathrm{ee}=74 \%, \mathrm{CHCl}_{3}\right)$.

${ }^{1} \mathrm{H}$ NMR (400 MHz, $\left.\mathrm{CDCl}_{3}\right): \delta=7.85-7.83(\mathrm{~m}, 4 \mathrm{H}), 7.50-7.45(\mathrm{~m}, 3 \mathrm{H}), 5.35-5.31(\mathrm{~m}, 1 \mathrm{H})$, 3.39-3.38 (m, 1H), 2.96-2.93 (m, 2H), $2.21(\mathrm{~s}, 3 \mathrm{H})$. Other spectroscopic data are in agreement with published data. ${ }^{3}$
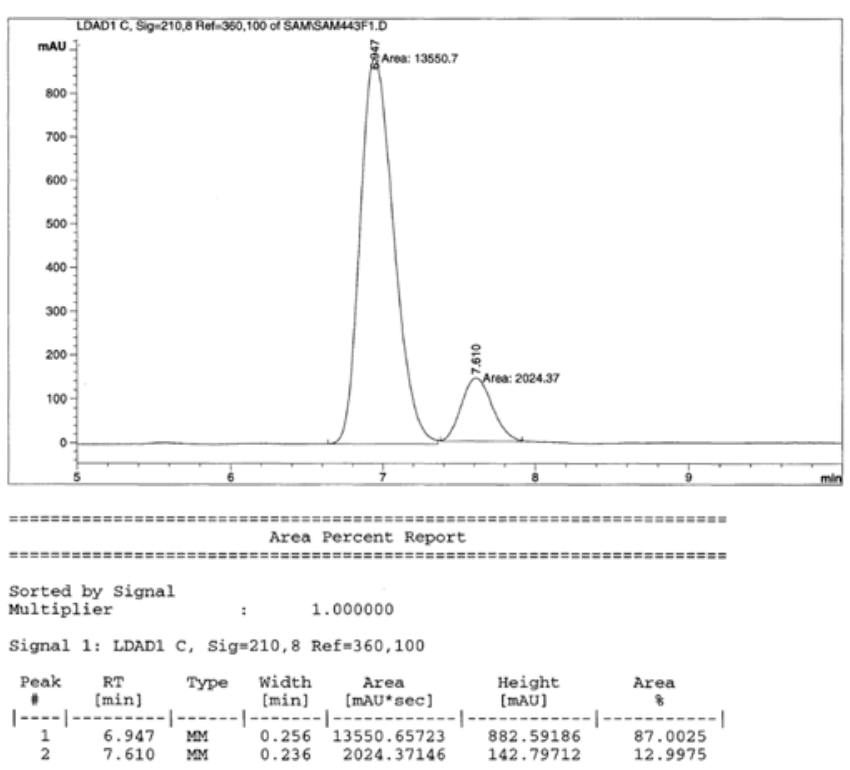


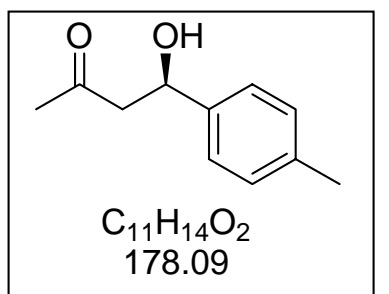

(R)-4-hydroxy-4-p-tolylbutan-2-one 3e (Table 2, entry 5): From ptolualdehyde $2 \mathbf{e}$ ( 1 eq., $0.3 \mathrm{mmol}, 35 \mu \mathrm{L}$ ) and L-proline (0.3 eq., 0.09 mmol, $10.3 \mathrm{mg})$ according to general procedure $1(15 \mathrm{~W}, 0$ bar, 32 ${ }^{\circ} \mathrm{C}$ ) to give $82 \%$ conversion (with $1: 1$ ratio of aldol product $3 \mathbf{e}$ and $\alpha, \beta$-unsaturated ketone derived from aldol product $3 \mathbf{e})$ after $1 \mathrm{~h}$ and to afford aldol product 3d (20.3 mg, 38\%). The enantiomeric excess was determined by chiral SFC (AS-H column, $2 \mathrm{~mL} / \mathrm{min}, 200$ bar, methanol 10\%-2-1-25\%, 30 ${ }^{\circ} \mathrm{C}, R_{\mathrm{t}}: 2.92(R), 3.24$ $(S)) \cdot[\alpha]_{\mathrm{D}}{ }^{20}=+21.4\left(\right.$ c $\left.0.41, \mathrm{ee}=64 \%, \mathrm{CHCl}_{3}\right)$.

${ }^{1} \mathrm{H}$ NMR $\left(400 \mathrm{MHz}, \mathrm{CDCl}_{3}\right): \delta=7.25-7.23(\mathrm{~m}, 2 \mathrm{H}), 7.17-7.15(\mathrm{~m}, 2 \mathrm{H}), 5.13-5.11(\mathrm{~m}, 1 \mathrm{H})$, $3.17(\mathrm{br}, 1 \mathrm{H}), 2.92-2.77(\mathrm{~m}, 2 \mathrm{H}), 2.33(\mathrm{~s}, 3 \mathrm{H}), 2.19(\mathrm{~s}, 3 \mathrm{H})$. Other spectroscopic data are in agreement with published data. ${ }^{5}$

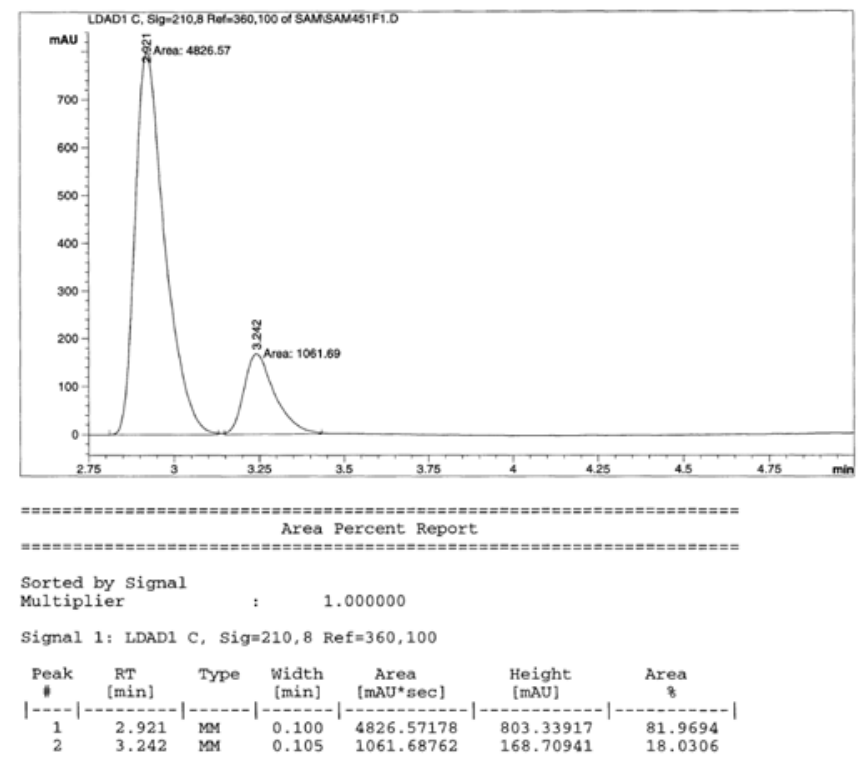

\footnotetext{
${ }^{5}$ Tang, Z.; Jiang, F.; Yu, L-T.; Cui, X.; Gong, L-Z.; Mi, A-Q.; Jiang, Y-Z.; Wu, Y-D. J. Am. Chem. Soc. 2003,
} 25,5262 . 


\section{Conjugate Additions}

General procedure 2 under microwave irradiation. In a $2-5 \mathrm{~mL}$ vessel were introduced $(R, R)$-iPBP (0.15 eq. $0.05 \mathrm{mmol}, 9.1 \mathrm{mg}), \mathrm{CHCl}_{3}(2.5 \mathrm{~mL}), \beta$-nitrostyrene (1 eq., 0.335 $\mathrm{mmol}, 50 \mathrm{mg}$ ) and finally the aldehyde or the ketone $(10 \mathrm{eq} ., 3.35 \mathrm{mmol})$ and the vessel was sealed with a septum and placed into the microwave cavity. The microwave source was then turned on. Constant microwave irradiation as well as simultaneous air-cooling ( 5 bar) were used during the entire reaction time. The evolution of the reaction was monitored by TLC. After cooling to room temperature, the mixture was then hydrolyzed as described in literature procedure ${ }^{1}$ using $1 \mathrm{~N} \mathrm{HCl}(2 \mathrm{~mL})$. The layers were separated and the aqueous phase was extracted with $\mathrm{CH}_{2} \mathrm{Cl}_{2}(2 \mathrm{x})$. The combined organic layers were dried over $\mathrm{MgSO}_{4}$, filtered, concentrated under reduced pressure and purified by flash column chromatography on silica gel using a mixture of cyclohexane and ethyl acetate.

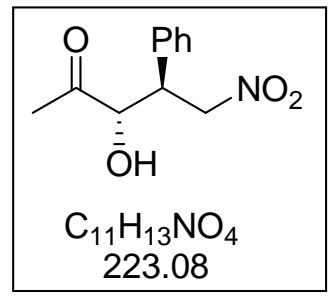

(3S,4S)-3-hydroxy-5-nitro-4-phenylpentan-2-one 6a (Table 3, entry 2) : From hydroxyketone 4a (10 eq., $3.35 \mathrm{mmol}, 0.23 \mathrm{~mL}$ ) according to general procedure $2\left(15 \mathrm{~W}, 0\right.$ bar, $\left.28^{\circ} \mathrm{C}\right)$ to give complete conversion after $4 \mathrm{~h}$ and to afford Michael adduct 6a as a mixture of two inseparable diastereoisomers (syn/anti 11:89) (62.3 mg, 83\%). The enantiomeric excess was determined by chiral SFC (OJ column, $2 \mathrm{~mL} / \mathrm{min}$, 200 bar, methanol $\left.5 \%-2-1-15 \%, 30^{\circ} \mathrm{C}, R_{\mathrm{t}}: 4.7(S, S), 5.5(R, R)\right)$.

${ }^{1} \mathrm{H}$ NMR $\left(400 \mathrm{MHz} \mathrm{CDCl}_{3}\right): \delta=7.41-7.23(\mathrm{~m}, 5 \mathrm{H}), 4.84-4.79\left(\mathrm{dd}, \mathrm{J}_{1}=6.04, \mathrm{~J}_{2}=13.36 \mathrm{~Hz}\right.$, $1 \mathrm{H}), 4.67-4.62\left(\mathrm{dd}, \mathrm{J}_{1}=8.32, \mathrm{~J}_{2}=13.64 \mathrm{~Hz}, 1 \mathrm{H}\right), 4.40-4.39(\mathrm{~m}, 1 \mathrm{H}), 3.83\left(\mathrm{dt}, \mathrm{J}_{1}=6.04, \mathrm{~J}_{2}=8.32\right.$ $\mathrm{Hz}, 1 \mathrm{H}), 3.80-3.73(\mathrm{~m}, 1 \mathrm{H}), 2.07(\mathrm{~s}, 3 \mathrm{H})$. Other spectroscopic data are in agreement with published data. ${ }^{1}$ 

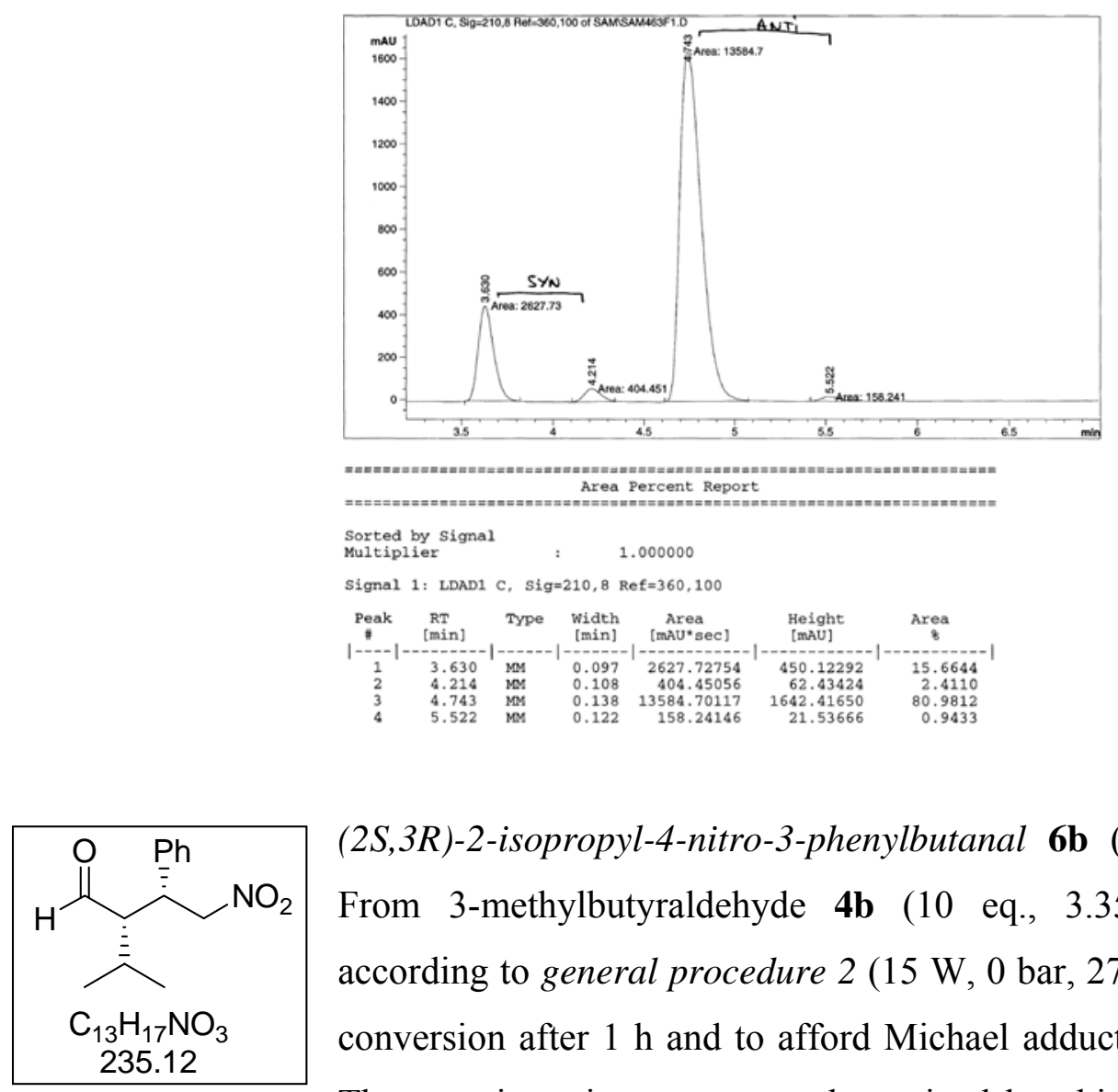

(2S,3R)-2-isopropyl-4-nitro-3-phenylbutanal 6b (Table 3, entry 4) : From 3-methylbutyraldehyde $4 \mathbf{b}$ (10 eq., $3.35 \mathrm{mmol}, 0.36 \mathrm{~mL})$ according to general procedure $2\left(15 \mathrm{~W}, 0 \mathrm{bar}, 27^{\circ} \mathrm{C}\right)$ to give complete conversion after $1 \mathrm{~h}$ and to afford Michael adduct $\mathbf{6 b}$ (76.4 mg, 97\%). The enantiomeric excess was determined by chiral GC (Hydrodex-B$3 \mathrm{P}$, iso $\left.150^{\circ} \mathrm{C}, R_{\mathrm{t}}: 38.48(R, S), 39.61(S, R)\right)$. $[\alpha]_{\mathrm{D}}{ }^{20}=-51.6\left(c 1.0\right.$, ee $\left.=78 \%, \mathrm{CHCl}_{3}\right)$.

${ }^{1} \mathrm{H}$ NMR (400 MHz, $\mathrm{CDCl}_{3}$ ) : $\delta=9.93$ (d, 1H, J = 2.2 Hz), 7.36-7.29 (m, 3H), 7.20-7.17 (m, $2 \mathrm{H}), 4.69-4.64(\mathrm{dd}, 1 \mathrm{H}, \mathrm{J}=4.32 \mathrm{~Hz}, \mathrm{~J}=12.64 \mathrm{~Hz}), 4.60-4.54(\mathrm{~m}, 1 \mathrm{H}), 3.92-3.86(\mathrm{~m}, 1 \mathrm{H})$, 2.79-2.75 (m, 1H), 1.75-1.67 (m, 1H), 1.10-1.09 (d, 3H, J= 7.08 Hz), 0.89-0.87 (d, 3H, J=7.08 $\mathrm{Hz})$.Other spectroscopic data are in agreement with published data. ${ }^{1}$

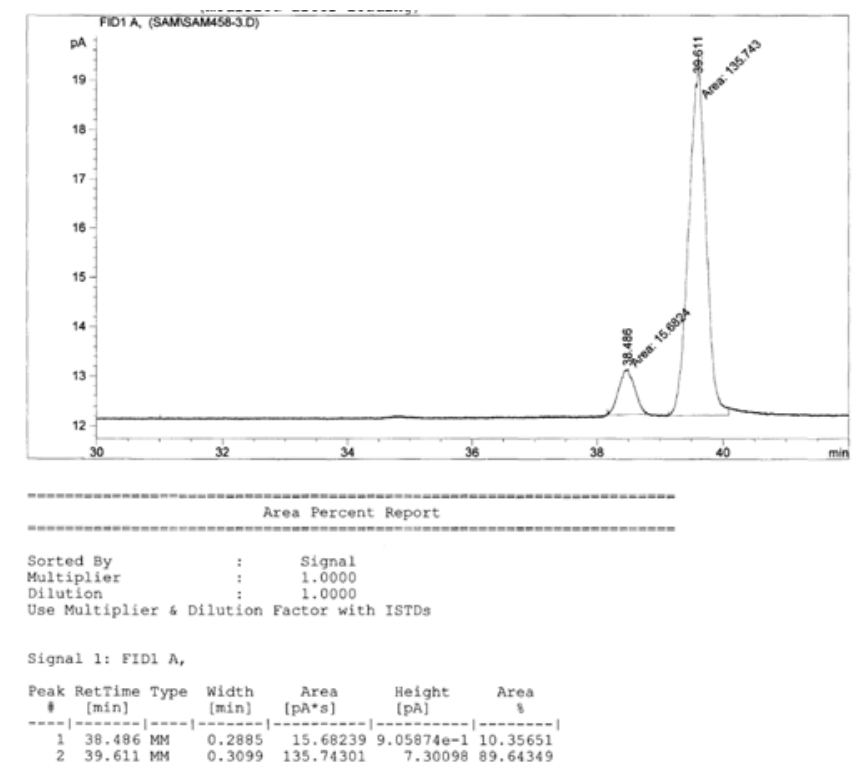




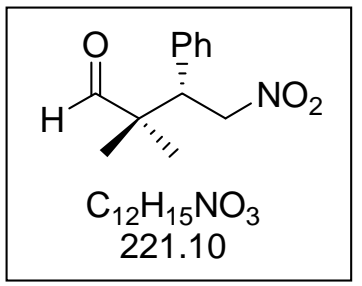

(S)-2,2-dimethyl-4-nitro-3-phenylbutanal 6c (Table 3, entry 6) : From isobutyraldehyde $4 \mathbf{c}$ (10 eq., $3.35 \mathrm{mmol}, 0.30 \mathrm{~mL}$ ) and according to general procedure $2\left(15 \mathrm{~W}, 0 \mathrm{bar}, 27^{\circ} \mathrm{C}\right)$ to give $80 \%$ conversion after $2 \mathrm{~h}$ and to afford Michael adduct 6c (56.7 mg, 76\%). The enantiomeric excess was determined by chiral GC (Hydrodex-B-3P, iso $140^{\circ} \mathrm{C}, R_{\mathrm{t}}: 47.39(R), 48.44(S)$ ). $[\alpha]_{\mathrm{D}}^{20}=-6.7\left(c 1.0, \mathrm{ee}=77 \%, \mathrm{CHCl}_{3}\right)$.

${ }^{1} \mathrm{H}$ NMR (400 MHz, $\left.\mathrm{CDCl}_{3}\right): \delta=9.53(\mathrm{~s}, 1 \mathrm{H}), 7.33-7.29(\mathrm{~m}, 3 \mathrm{H}), 7.20-7.18(\mathrm{~m}, 2 \mathrm{H}), 4.88-$ $4.82(\mathrm{~m}, 1 \mathrm{H}), 4.71-4.66(\mathrm{~m}, 1 \mathrm{H}), 3.80(\mathrm{~m}, 1 \mathrm{H}), 1.13(\mathrm{~s}, 3 \mathrm{H}), 1.00(\mathrm{~s}, 3 \mathrm{H})$. Other spectroscopic data are in agreement with published data. ${ }^{1}$

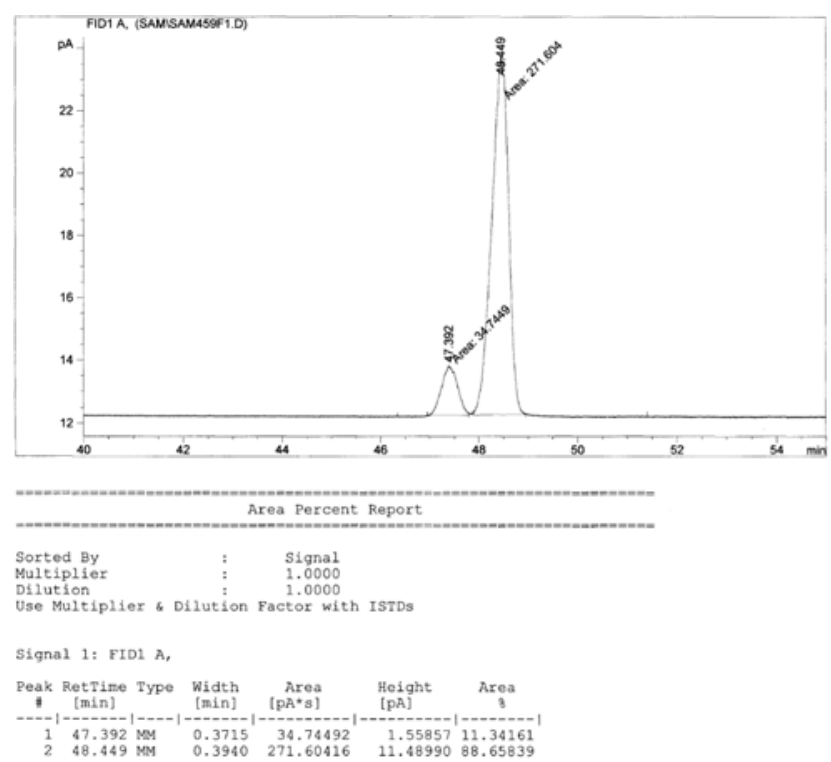

\section{Diels-Alder reaction}

General procedure 3 under microwave irradiation. In a $2-5 \mathrm{~mL}$ vessel were introduced (5S)-5-Benzyl-2,2,3-trimethylimidazolidin-4-one hydrochloride 9 (0.05 eq. 0.095 mmol, 25 $\mathrm{mg}), \mathrm{MeOH} / \mathrm{H}_{2} \mathrm{O}(95 / 5 \mathrm{v} / \mathrm{v} 2 \mathrm{~mL})$, and $(E)$-cinnamaldehyde 8 (1 eq., $1.915 \mathrm{mmol}, 0.25 \mathrm{~mL}$ ). The solution was stirred 2 minutes under argon before addition of cyclopentadiene monomer 7 (3 eq., $5.745 \mathrm{mmol}, 0.48 \mathrm{~mL}$ ) and the vessel was sealed with a septum and placed into the microwave cavity. The microwave source was then turned on. Constant microwave irradiation as well as simultaneous air-cooling ( 5 bar) were used during the entire reaction time. The evolution of the reaction was monitored by TLC. After cooling to room temperature, the mixture was then quenched as described in literature procedure ${ }^{2}$ diluting with $\mathrm{Et}_{2} \mathrm{O}$ and washed successively with $\mathrm{H}_{2} \mathrm{O}$ and brine. The organic layer was dried over $\mathrm{Na}_{2} \mathrm{SO}_{4}$, filtered, 
concentrated under reduced pressure. Hydrolysis of the product dimethyl acetal was performed by stirring the crude product mixture in TFA: $\mathrm{H}_{2} \mathrm{O}: \mathrm{CHCl}_{3}(1: 1: 2,12 \mathrm{~mL})$ for $2 \mathrm{~h}$ at room temperature, followed by neutralization with sat. aq. $\mathrm{NaHCO}_{3}$ and extraction with $\mathrm{Et}_{2} \mathrm{O}$. Purification of the Diels-Alder adduct was accomplished by silica gel chromatography using a mixture of cyclohexane and ethyl acetate.

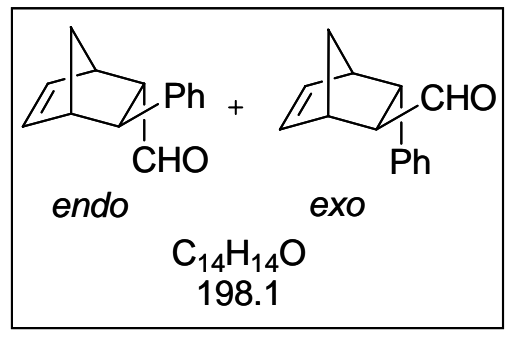

$(1 S, 2 S, 3 S, 4 R)-3$-phenylbicyclo[2.2.1]hex-5-ene-2carboxaldehyde endo-10 and (1R,2S,3S,4S)-3phenylbicyclo[2.2.1]hex-5-ene-2-carboxaldehyde exo-10 (Table 4, entry 3) : Prepared according to general procedure $3\left(50 \mathrm{~W}, 1 \mathrm{bar}, 65^{\circ} \mathrm{C}\right)$ to give $84 \%$ conversion after $1 \mathrm{~h}$ and to afford 1:1.2 endo-10/exo-10 mixture as a pale yellow oil. Product ratio was determined by ${ }^{1} \mathrm{H}$ NMR on the crude material. The enantiomeric excess of each diatereoisomer was determined on the primary alcohols coming from the reduction of the aldehydes endo-10/exo10 mixture by SFC (chiralpak AD column, $2 \mathrm{~mL} / \mathrm{min}, 200$ bar, $\mathrm{MeOH} 10 \%-2-1-25 \%, 30^{\circ} \mathrm{C}$, $R_{\mathrm{t}}$ : exo : $4.80(R), 4.5 .51(S)$, and endo : $\left.6.64(R), 7.90(S)\right)$.

Endo-10 : ${ }^{1} \mathrm{H}$ NMR (400 MHz, $\left.\mathrm{CDCl}_{3}\right): \delta=9.61(\mathrm{~d}, 1 \mathrm{H}, \mathrm{J}=), 7.14-7.33(\mathrm{~m}, 5 \mathrm{H}), 6.62-6.43$ (m, 1H), 6.17-6.15 (m, 1H), 3.34 (br, 1H), 3.13 (br, 1H), 3.09 (m, 1H), 2.99 (m, 1H), 1.79$1.84(\mathrm{~m}, 1 \mathrm{H}), 1.65-1.59(\mathrm{~m}, 1 \mathrm{H})$. Other spectroscopic data are in agreement with published data. $^{6}$

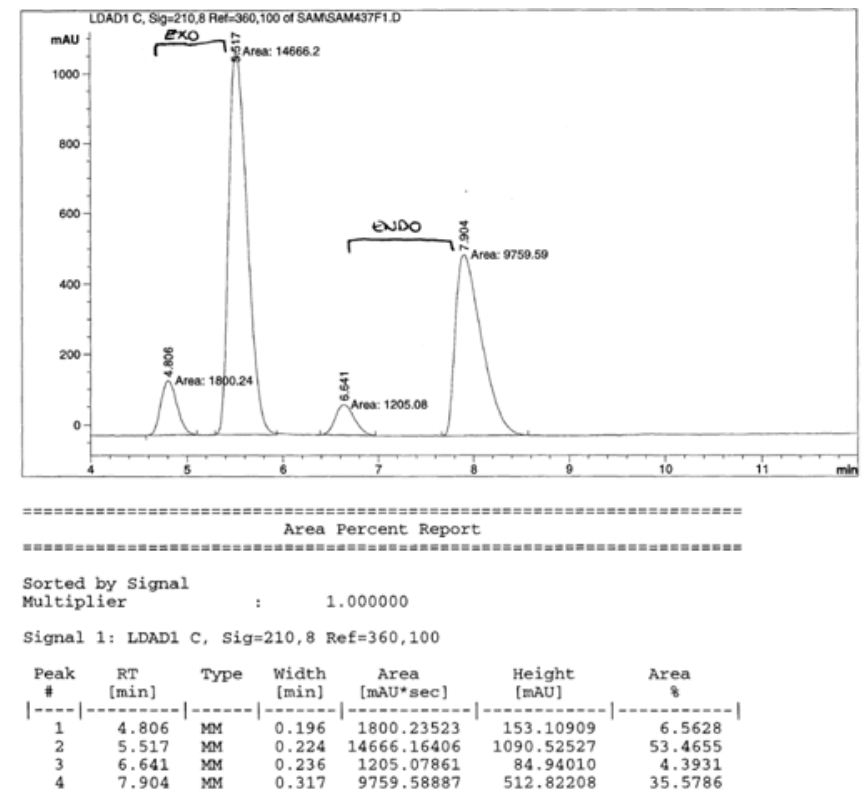

\footnotetext{
${ }^{6}$ Ishihara, K.; Kurihara, H.; Matsumoto, M.; Yamamoto, H. J. Am. Chem. Soc. 1998, 120, 6920.
} 

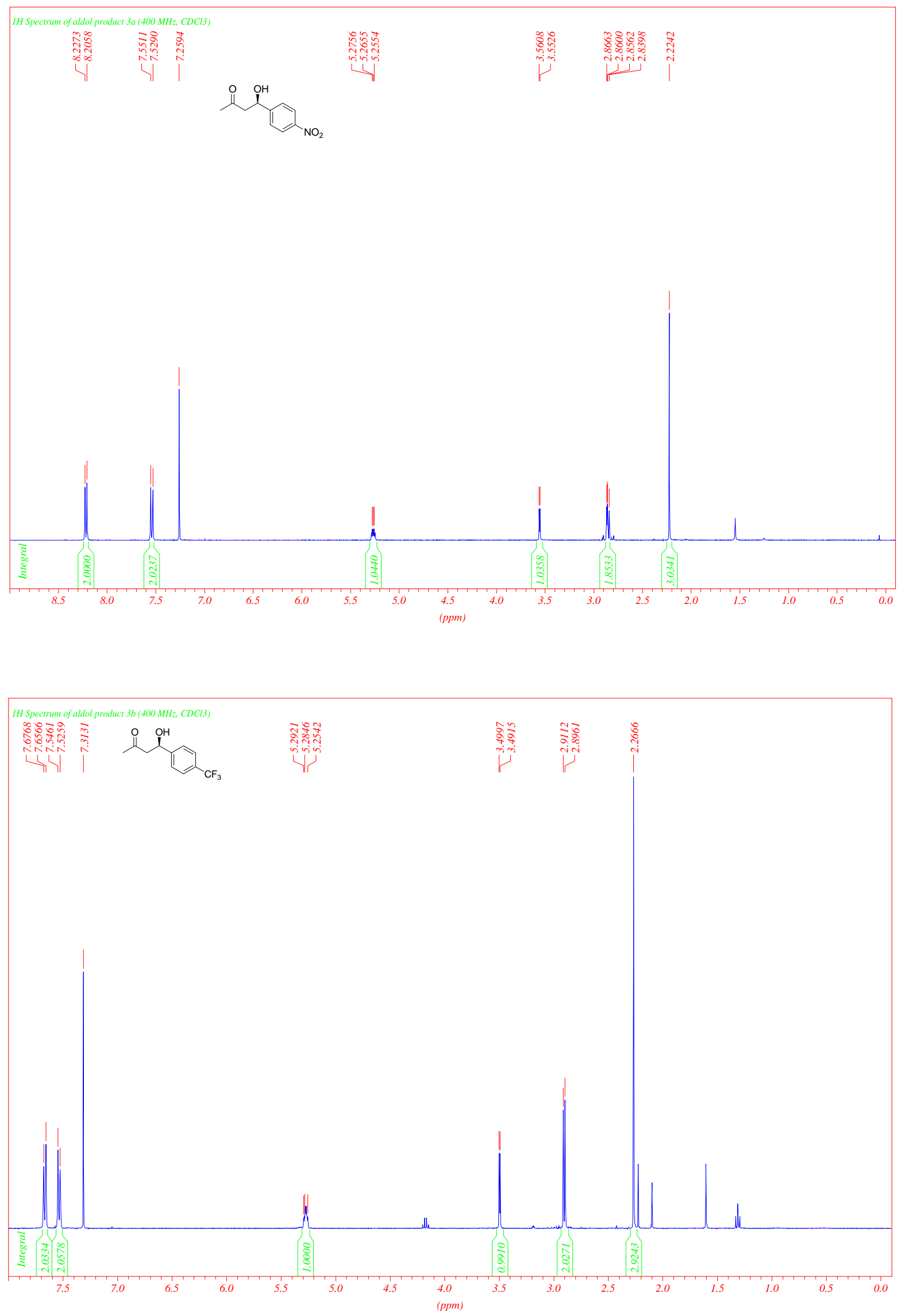

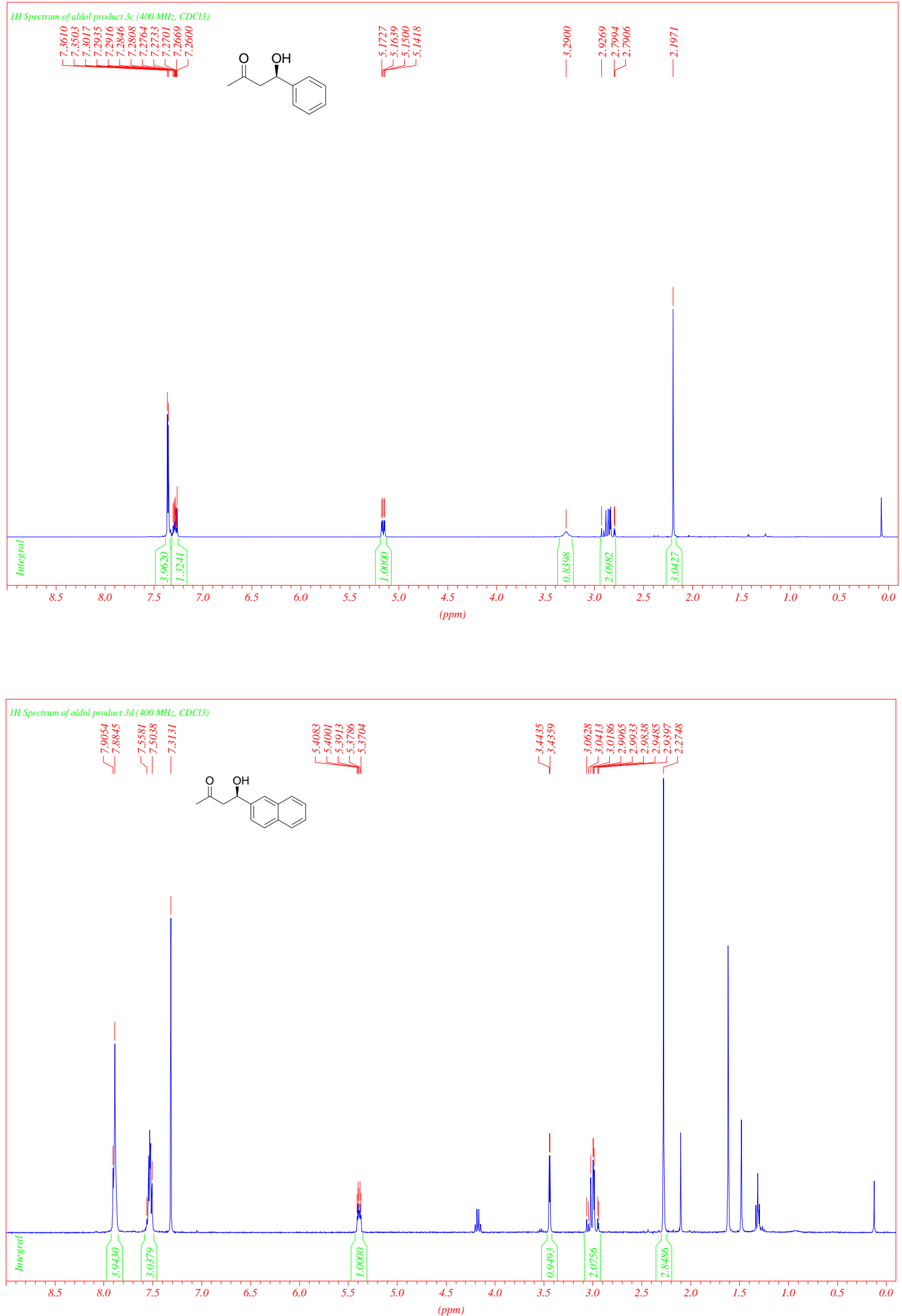

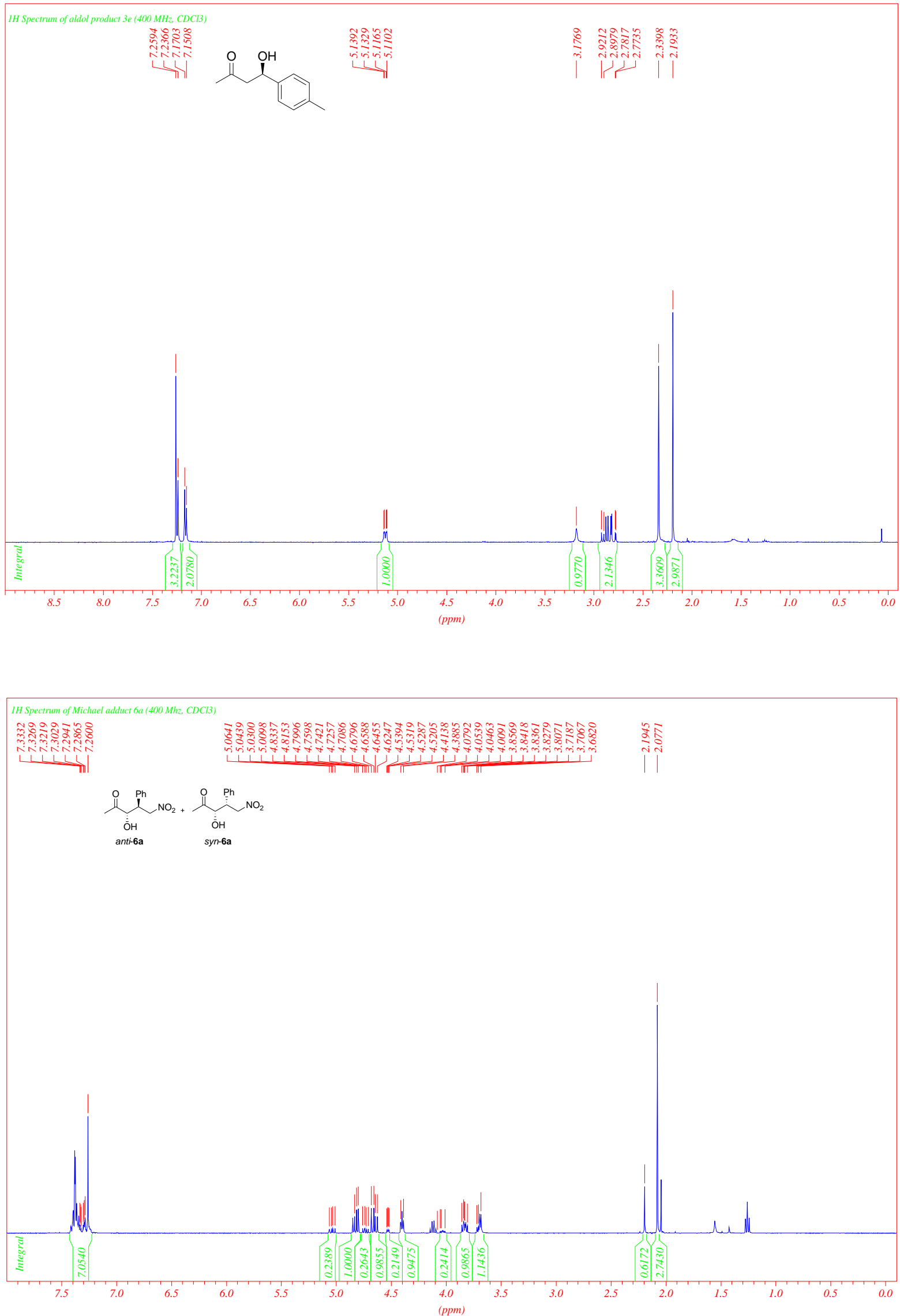

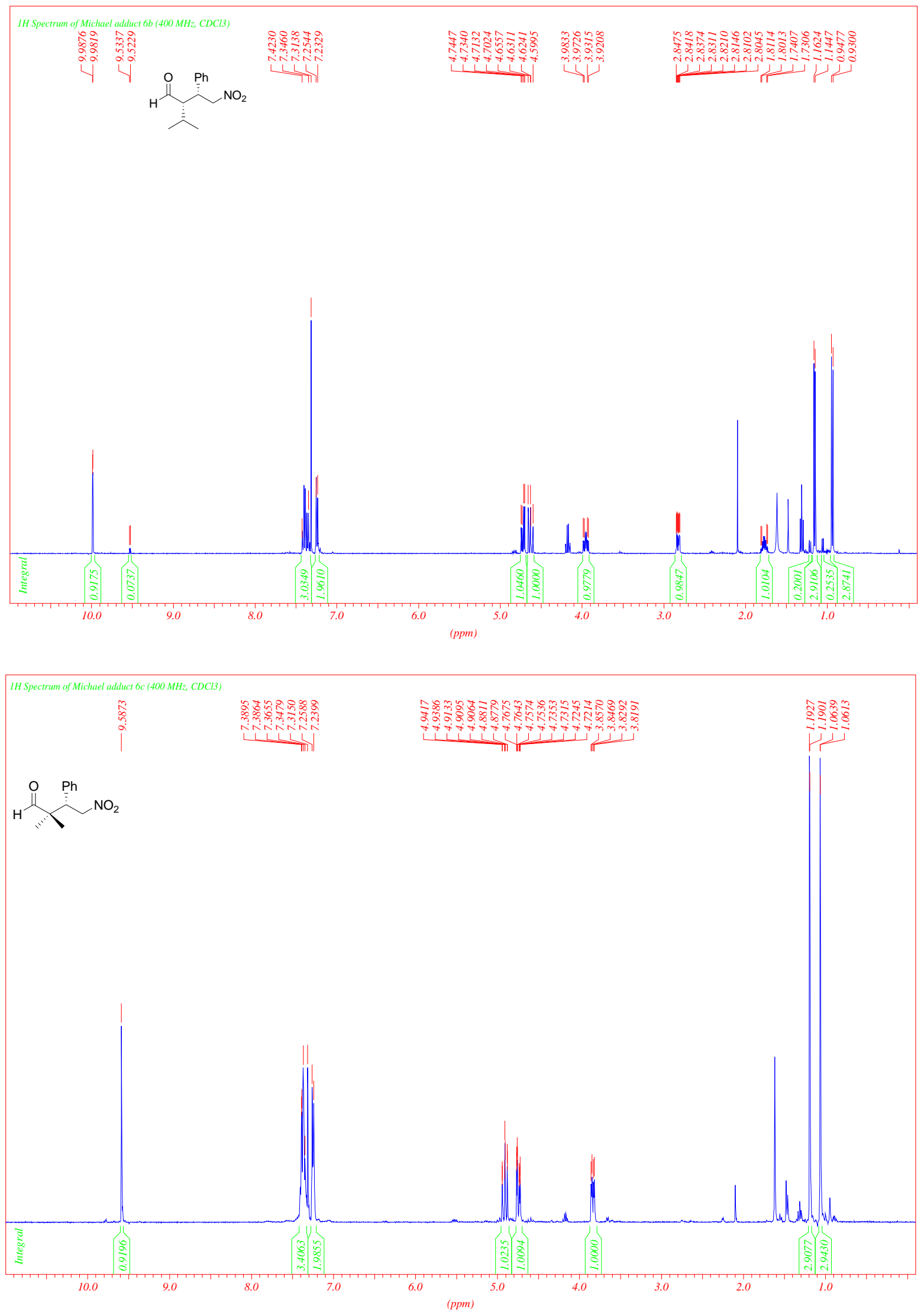


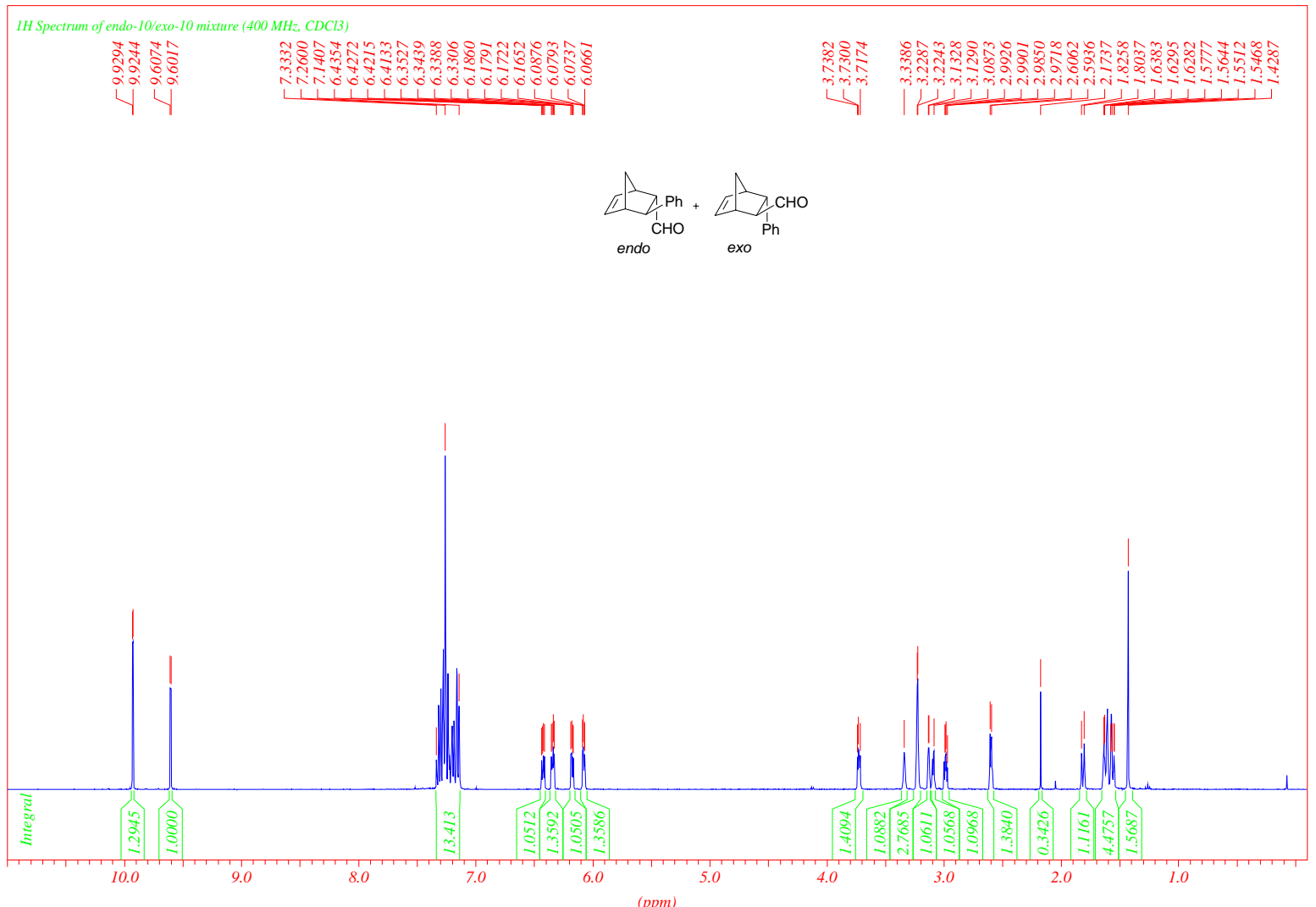


Temperature, pressure and microwave power profiles for the reaction mixture corresponding to Table 1, entry 10 (constant microwave power of $15 \mathrm{~W}$ during $15 \mathrm{~min}$, using simultaneous cooling).

Status: OK

Absorption Level: Very High

Vial: 2.0-5.0 ml

Pre-stirring: 0

Initial power: 0

Step Time $\quad{ }^{\circ} \mathrm{C}$ bar W FHT Cooling

1 00:15:00 Off Off 15 On On

Temperature $\left({ }^{\circ} \mathrm{C}\right)$

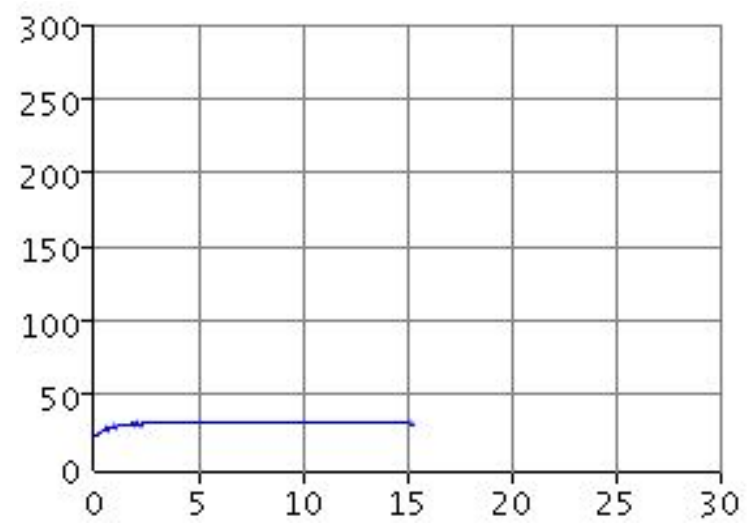

Pressure (bar)

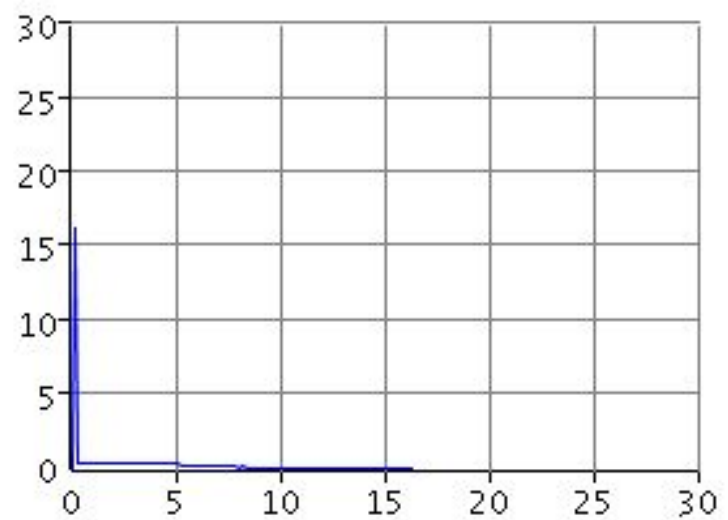

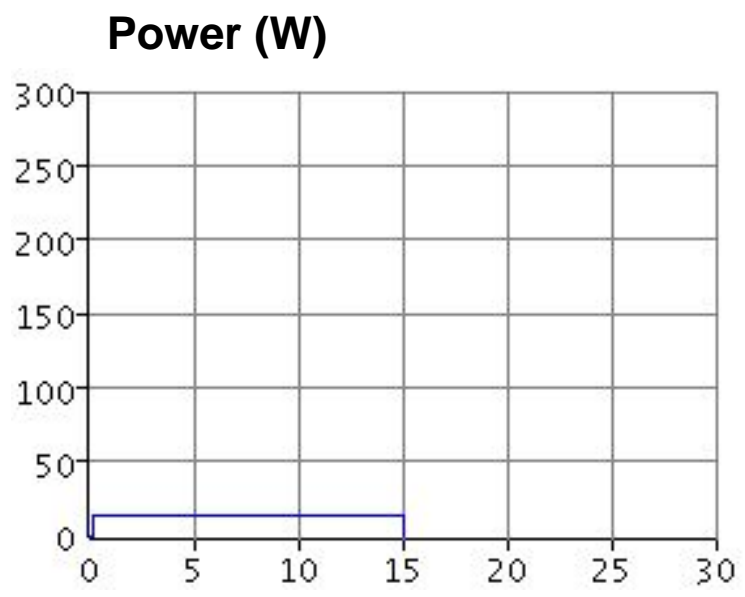


Temperature, pressure and microwave power profiles for the reaction mixture corresponding to Table 2, entry 1(constant microwave power of $15 \mathrm{~W}$ during 15 min, using simultaneous cooling).

Status: OK

Absorption Level: Very High

Vial: $2.0-5.0 \mathrm{ml}$

Pre-stirring: 0

Initial power: 0

\begin{tabular}{|c|c|c|}
\hline Step & Time & \begin{tabular}{l|l|l}
${ }^{\circ} \mathrm{C}$ & bar W & FHT Cooling
\end{tabular} \\
\hline & $0.15 \cdot 00$ & Off Off 15 On \\
\hline
\end{tabular}

Temperature $\left({ }^{\circ} \mathrm{C}\right)$

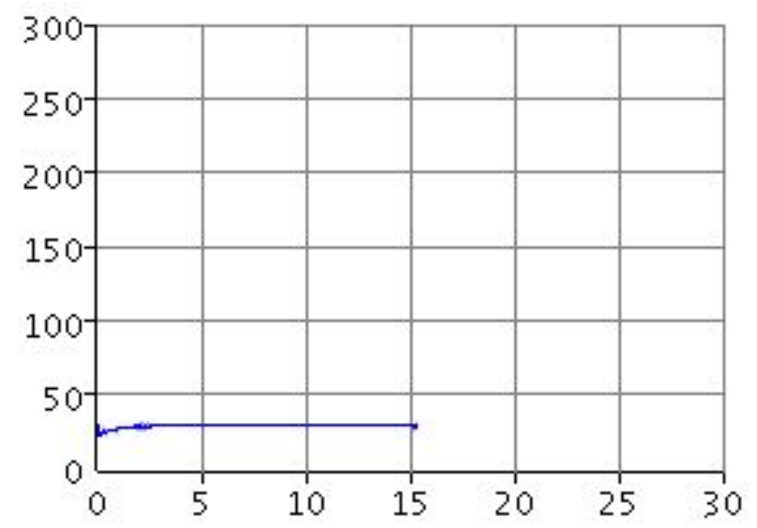

Pressure (bar)

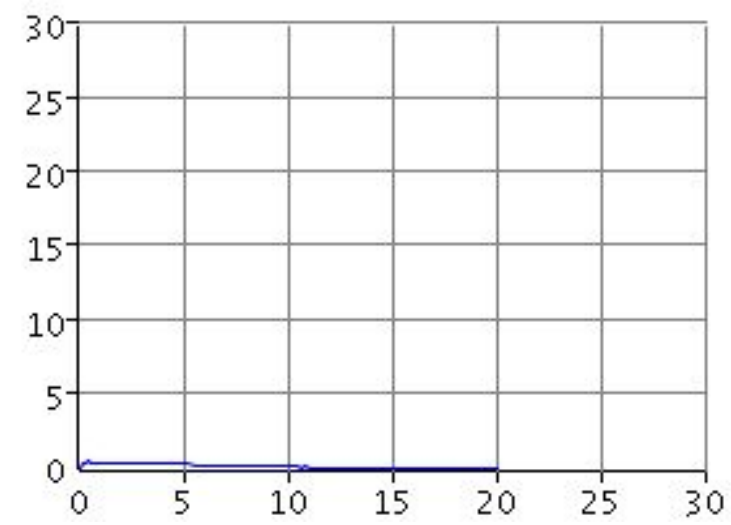

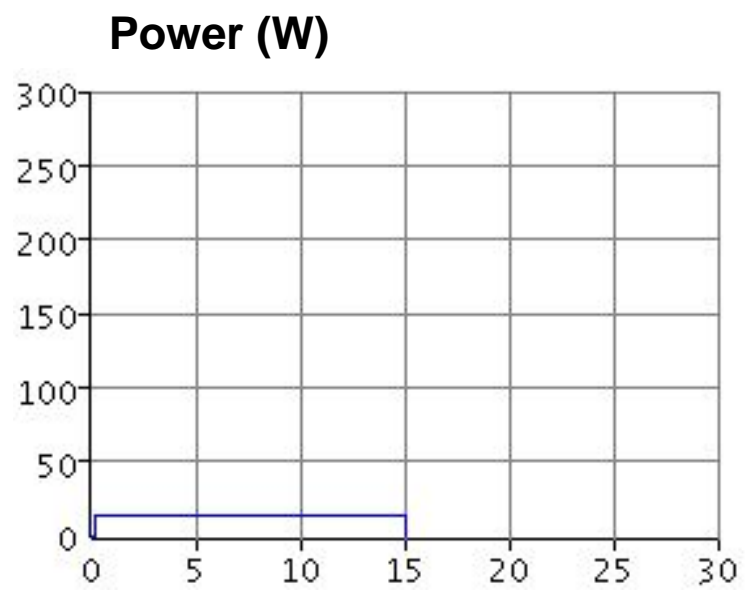


Temperature, pressure and microwave power profiles for the reaction mixture corresponding to Table 2, entry 3 (constant microwave power of $15 \mathrm{~W}$ during $1 \mathrm{~h}$, using simultaneous cooling).

Status: OK

Absorption Level: Very High

Vial: 2.0-5.0 ml

Pre-stirring: 0

Initial power: 0

\begin{tabular}{|c|c|c|}
\hline Step & Time & ${ }^{\circ} \mathrm{C}$ bar W FHT Cooling \\
\hline & 1. $1 \cdot 0$ & Off Off $15 \mathrm{On}$ \\
\hline
\end{tabular}

Temperature $\left({ }^{\circ} \mathrm{C}\right)$

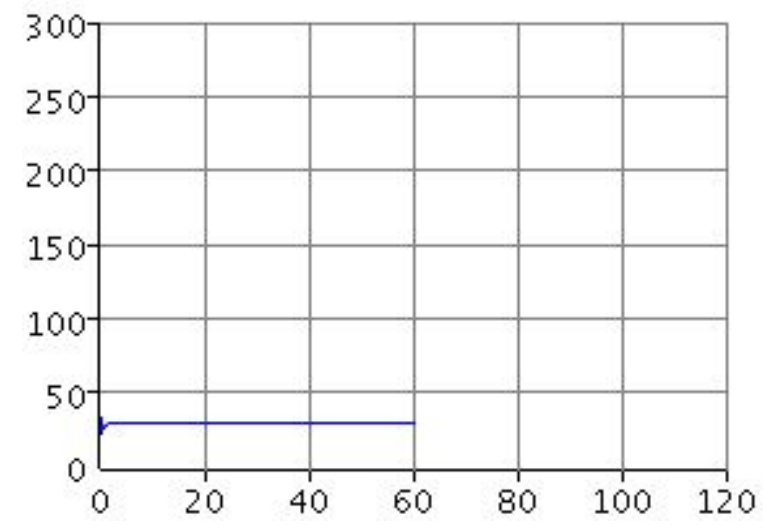

Pressure (bar)

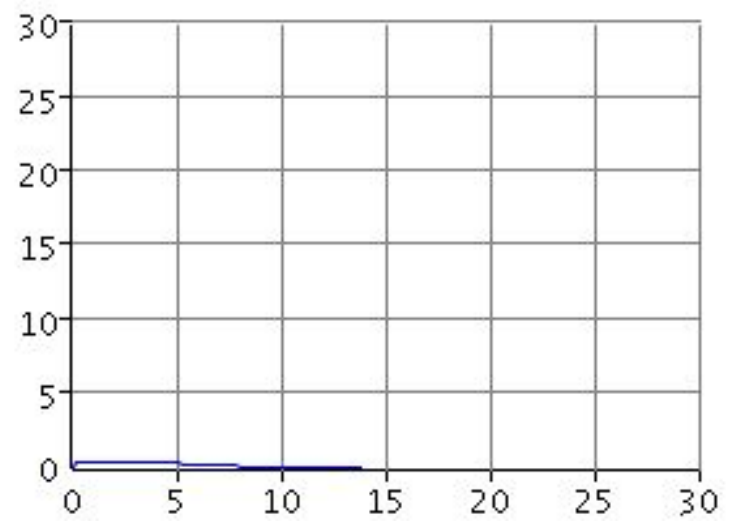

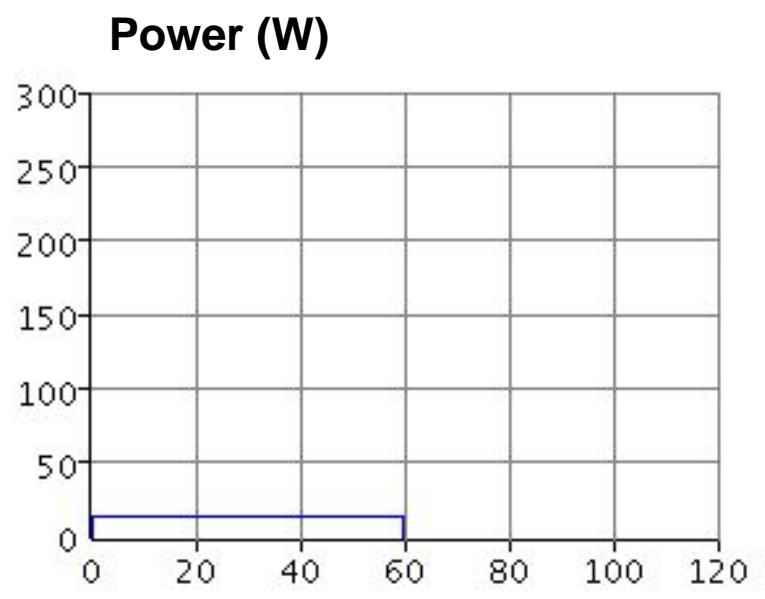

